\title{
Calcium Restores Prepenetration Morphogenesis Abolished by Methylglyoxal-Bis-Guanyl Hydrazone in Cochliobolus miyabeanus Infecting Rice
}

\author{
Il-Pyung Ahn and Seok-Cheol Suh
}

National Institute of Agricultural Biotechnology, Rural Development Administration, Suwon 441-100, Korea. Accepted for publication 22 September 2006.

\section{ABSTRACT}

Ahn, I.-P., and Suh, S.-C. 2007. Calcium restores prepenetration morphogenesis abolished by methylglyoxal-bis-guanyl hydrazone in Cochliobolus miyabeanus infecting rice. Phytopathology 97:331-337.

Cochliobolus miyabeanus forms a specialized infection structure, an appressorium, to infect its host rice plants. Curtailment of prepenetration development by spermidine and spermine was more evident in appressorium development and germination remained unaffected, whereas putrescine and methylglyoxal-bis-guanyl hydrazone (MGBG) impaired both morphogenetic events. Exogenous calcium nullified the inhibitory effect of MGBG on the prepenetration development in vitro and in vivo and the disease progression. High levels of polyamines were detected in freshly collected conidia, but the amounts were reduced during germination and appressorium formation. MGBG fortified the decrease of polyamines within conidia under development and calcium amendment did not affect the reduction. Hard-surface contact augmented messenger RNA synthesis of calmodulin gene $(\mathrm{CmCaM})$ and protein kinase $\mathrm{C}(\mathrm{PKC})$ activity in germinating or appressorium-forming conidia. Calcium restored transcription of $\mathrm{CmCaM}$ and upregulation of PKC activity suppressed by MGBG. Taken together, fine-tuning of intracellular polyamine transition is indispensable for the conidial germination and appressorium formation in C. miyabeanus. Biochemical and molecular analyses revealed that the MGBG-acting site or sites are upstream of $\mathrm{Ca}^{2+}$-dependent signaling pathways regulating prepenetration morphogenesis of C. miyabeanus causing rice brown leaf spot.

Additional keywords: Bipolaris oryzae, Oryza sativa.
Cochliobolus miyabeanus (Ito \& Kuribayashi in Ito) Drechs. ex Dastur, the teleomorph of Bipolaris oryzae (Breda de Haan) Shoemaker, is a necrotrophic fungal pathogen that causes brown leaf spot, one of the most devastating and prevalent diseases of rice (Oryza sativa L.). In 1943, an epidemic of the disease caused the Bengal famine and was a major factor causing two million fatalities (39). An outbreak of the disease on rice flag leaves often results in severe yield losses. Control of the disease has been dependent on synthetic fungicides; however, recent emergence of resistant isolates (27) and environmental regulations have restricted the use of fungicides. One of the alternatives is the introduction of target site-specific chemicals preventing fungal infection.

Early infection processes of this genus include conidial attachment to the host surface, appressorium formation, and direct penetration of host cuticle using an infection hypha differentiated from the appressorium (33). In addition, in the last decade, several works showed the prepenetration morphogenesis of Cochliobolus spp. and characterized some fungal genes involved in these developments $(7,10,16,21)$. Disruption of G-protein $\alpha$ and $\beta$ subunits of $C$. heterostrophus resulted in the inhibition of appressorium formation $(12,16)$. In contrast with the results from the $\alpha$ subunit deletion, disruption of the $\beta$ subunit resulted in the reduction of fungal virulence on maize leaves $(16,21)$. Synchronous inhibition of appressorial development and disease implies that conidial germination and appressorium formation should be indispensable for the infection to take place and ubiquitous signal

Corresponding author: I.-P. Ahn; E-mail address: jinhyung@ rda.go.kr

* The $\boldsymbol{e}$-Xtra logo stands for "electronic extra" and indicates that Figures 2 and 3 appear in color online.

DOI: 10.1094/PHYTO-97-3-033

(C) 2007 The American Phytopathological Society transduction cascades might participate in these morphogenetic events of Cochliobolus spp.

Polyamines (putrescine, spermidine, and spermine) are common and evolutionarily ancient organic cations of low molecular weight that influence numerous cellular processes, involving transcription and chromatin structure $(32,35)$, and essential for plant growth (3) and cell development (40). Many microorganisms and higher plants are able to produce polyamines from arginine and ornithine, whereas most plant-pathogenic fungi use ornithine as the sole polyamine source (42). Therefore, inhibitors of ornithine decarboxylase (ODCase), such as difluoromethylornithine (DFMO), have been developed to inhibit human and plantpathogenic fungi through disturbance of the fungal polyamine metabolism (23). Polyamine biosynthesis inhibitors interfere with several plant diseases $(23,36,43)$. In addition, disruption of ODCase, required for the synthesis of putrescine in fungi, resulted in the near abolishment of virulence in Stagonospora nodorum (5) and Ustilago maydis (19). Reitz et al. (37) showed that treatment of methylglyoxal-bis-guanyl hydrazone (MGBG), a potent inhibitor of S-adenosylmethionine (SAM) decarboxylase, impairs appressorium formation in Uromyces viciae-fabae. Inhibitors of polyamine biosynthesis also interfere with sclerotial formation in Sclerotinia sclerotiorum (34). These results evidently manifest that polyamines also participate in fungal development and pathogenicity. Furthermore, polyamine metabolism should be one of the target sites for developing novel fungicides.

Extensive studies using a wide range of pharmacological agents, which disrupt $\mathrm{Ca}^{2+}$ influxes or interfere with the calcium-binding protein, have strongly suggested the importance of $\mathrm{Ca}^{2+}$ signaling systems in the prepenetration morphogenesis of Colletotrichum spp. $(29,41,44)$ and Magnaporthe grisea, causing rice blast (30). Contacting hard surface and treatment with ethylene, both physicochemical host-mimicking signals, trigger boosted transcription of 
calmodulin gene in germinating or appressorium-forming conidia of Colletotrichum gloeosporioides infecting avocado (29). Furthermore, exogenous calcium restores prepenetration morphogenesis and mRNA synthesis of calmodulin gene inhibited by polyamines in C. gloeosporioides pathogenic on red pepper (1). These results indicate that calcium- or calmodulin-dependent signal transduction participates in prepenetration events in these fungi.

Many of the protein kinase C (PKC) target substrates are constituents of signal transduction pathways and include proteins that regulate ion channels, calcium- and calmodulin-binding proteins, growth factor receptors, structural and regulatory proteins of the cytoskeleton, components of the transcriptional machinery, efflux pumps, and many other proteins $(4,24)$. Signal transduction mediated by PKC is essential for the conservation of cellular integrity and normal development in Saccharomyces cerevisiae and PKC extends its activity through regulation of mitogenactivated protein (MAP) kinase cascades (20).

The purpose of this study was to investigate the effects of polyamines and MGBG on the prepenetration morphogenesis of Cochliobolus miyabeanus in vitro and in vivo. In addition, biochemical and molecular analyses were performed to explain how exogenous calcium nullifies inhibitory effects of MGBG on the conidial germination and appressorium formation and on the progression of rice brown leaf spot.

\section{MATERIALS AND METHODS}

Fungal strains. C. miyabeanus strain $\mathrm{HIH}-1$ was isolated from symptomatic rice seed. Conidia were harvested from 1-week-old cultures grown on sucrose proline agar (SPA) (13) at $22^{\circ} \mathrm{C}$ under the continuous fluorescent light. Fungal stock was preserved as a conidial suspension amended with $20 \%$ glycerol at $-70^{\circ} \mathrm{C}$ until use.

Cell development and chemical treatments. Germination and appressorium formation assays were performed as previously described (2). Polyamines (putrescine, spermidine, and spermine) and MGBG, an inhibitor of SAM decarboxylase, were purchased from Sigma-Aldrich (St. Louis, USA). Cyclic AMP and 1,16hexadecanediol (cutin monomer) were purchased from Fluka (Buchs, Switzerland). Drops $(45 \mu \mathrm{l})$ of conidial suspension $(1 \times$ $10^{5}$ conidia $\left./ \mathrm{ml}\right)$ were placed on the hydrophobic surface of GelBond (FMC Corp., Rockland, ME, USA) and $5 \mu$ of chemical solutions to be examined were supplemented. Conidial germination and appressorium formation were measured $12 \mathrm{~h}$ after incubation at $22^{\circ} \mathrm{C}$. All experiments were conducted more than five times with three replicates.

Assay of appressorium on detached rice leaves. Pieces $(\approx 5 \times$ $2 \mathrm{~cm}^{2}$ ) of a rice (cv. Nakdong) leaf were attached to a slide glass with cellophane tape and placed in a moistened petri dish. Three drops $(20 \mu \mathrm{l})$ of a conidial suspension $\left(1 \times 10^{4}\right.$ conidia $\left./ \mathrm{ml}\right)$ with mock (Tween 20 at $250 \mu \mathrm{g} / \mathrm{ml}$ ), $1 \mathrm{mM} \mathrm{MGBG}$, and $1 \mathrm{mM} \mathrm{CaCl}_{2}$, with $1 \mathrm{mM} \mathrm{MGBG}$ and $1 \mathrm{mM} \mathrm{CaCl}$, were placed on the leaf pieces and incubated for $12 \mathrm{~h}$ at $25^{\circ} \mathrm{C}$. Pieces of inoculated rice leaves were cut and fixed with lactophenol for $16 \mathrm{~h}$. After fixation, chlorophyll was removed by immersing the leaf sections twice in the mixture of lactophenol and ethanol $(1: 1)$ for $2 \mathrm{~h}$ at $95^{\circ} \mathrm{C}$. After staining with aniline blue $(0.01 \%$ in a mixture of equal volume of ethanol, lactic acid, and phenol) at $25^{\circ} \mathrm{C}$ for at least $24 \mathrm{~h}$, the numbers of germinating or appressorium-forming conidia were measured by microscopic observation. This experiment was repeated three times with three replicates.

Effect of MGBG on rice brown leaf spot. Rice plants (cv. Nakdong) were grown in commercial soil mix in plastic pots ( $5 \mathrm{~cm}$ in diameter) for 4 weeks in the greenhouse. Mock (Tween 20 at $250 \mu \mathrm{g} / \mathrm{ml})$ or MGBG $(1 \mathrm{mM}$, and Tween 20 at $250 \mu \mathrm{g} / \mathrm{ml})$ was sprayed on 10 rice plants at the fifth-leaf stage and air dried. After $24 \mathrm{~h}$, a $2 \times 10^{5}$ conidia/ml suspension of $C$. miyabeanus amended with mock or with $1 \mathrm{mM} \mathrm{CaCl}_{2}$ and Tween 20 at
$250 \mu \mathrm{g} / \mathrm{ml}$ was sprayed on the mock- or MGBG-treated rice plants, placed in a dew chamber $\left(25^{\circ} \mathrm{C}, 100 \%\right.$ relative humidity) for 16 to $24 \mathrm{~h}$, and transferred into a greenhouse. Disease progression was assessed 7 days after inoculation. Disease severity was determined using a scale of 0 to 9 , where $0=$ no symptoms, $1=<1 \%, 2=1$ to $3 \%, 3=4$ to $5 \%, 4=6$ to $10 \%, 5=11$ to $15 \%$, $6=16$ to $25 \%, 7=26$ to $50 \%, 8=51$ to $75 \%$, and $9=>76 \%$ of affected leaf area according to the method developed by the International Rice Research Institute (22). All experiments were repeated more than three times.

RNA protein preparation and Northern blot hybridization analysis. Conidia harvested from 1-week-old cultures were washed twice with ice-cold distilled water, and $7 \times 10^{7}$ conidia suspended in $300 \mathrm{ml}$ of water, in $1 \mathrm{mM} \mathrm{MGBG}$, in $1 \mathrm{mM} \mathrm{CaCl}_{2}$, and in a mixture of $1 \mathrm{mM} \mathrm{MGBG}$, and $1 \mathrm{mM} \mathrm{CaCl}_{2}$ were placed on each of $8035-\mathrm{cm}^{2}$ glass plates and incubated for $4 \mathrm{~h}$ at $23 \pm$ $2{ }^{\circ} \mathrm{C}$. After removing excessive moisture by blotting with a paper towel, germinating or appressorium-forming conidia were harvested by scraping them off the glass plate with a rubber policeman and pulverized with Geno/Grinder (SPEX CertiPrep, Metuchen, NJ). Total RNA was isolated as described and northern blot hybridization analysis were performed with ${ }^{32} \mathrm{P}$-labeled calmodulin complementary DNA ( $\mathrm{CmCaM}$; GenBank accession no. DQ471846) as the probe. Total fungal protein was extracted in ice-cold buffer $(20 \mathrm{mM}$ sodium phosphate [pH 7.6], $10 \mathrm{mM} \beta$ mercaptoethanol, $4 \mathrm{mM}$ EGTA, and $0.5 \mathrm{mM} \mathrm{PMSF}$ ) and the debris was removed by centrifugation at $5,000 \times g$ and $4{ }^{\circ} \mathrm{C}$ for 10 min. Quantification of total protein within the supernatants was performed by the Bradford method $(6,26)$.

Measurement of PKC activity. PKC activity within $10 \mu \mathrm{g}$ of total protein was measured by using a nonradioactive PKC assay system (Promega Corp., Madison, WI), following the manufacturer's instructions. This assay system uses colored and fluorescent $\mathrm{C} 1$ peptide (P-L-S-R-T-L-S-V-A-A-K, kemptide) as a substrate. Phosphorylation of $\mathrm{C} 1$ peptide by $\mathrm{PKC}$ alters the peptide's net charge from +1 to -1 . This change allows the phosphorylated and nonphosphorylated peptides to be separated by agarose gel electrophoresis at neutral $\mathrm{pH}$. Phosphorylated peptide (which migrates toward the positive electrode) was cut from agarose gel and kinase activity was quantified spectrophotometrically at $570 \mathrm{~nm}$ following the manufacturer's instructions. One unit of kinase is defined as the number of pmoles of phosphate transferred per minute to a substrate.

Extraction and quantification of polyamines. Polyamines were extracted from ground conidia $(100 \mathrm{mg})$ with $1 \mathrm{ml}$ of icecold 5\% perchloric acid. After extraction for an hour in an ice bath, debris was removed by centrifugation at $26,000 \times g$ and $4^{\circ} \mathrm{C}$ for $15 \mathrm{~min}$. The supernatant (perchloric acid soluble) was used for analysis of free polyamines (15). A $500-\mu \mathrm{l}$ portion was added to $1 \mathrm{ml}$ of $2 \mathrm{~N} \mathrm{NaOH}$, mixed with $10 \mu \mathrm{l}$ of benzoyl chloride, and agitated vigorously for $10 \mathrm{~s}$. Then, $2 \mathrm{ml}$ of saturated $\mathrm{NaCl}$ solution was added. After addition with $2 \mathrm{ml}$ of diethyl ether, the mixture was agitated vigorously for $10 \mathrm{~min}$ and centrifuged at $5,000 \times g$ and $4^{\circ} \mathrm{C}$ for $10 \mathrm{~min}$. A $1-\mathrm{ml}$ portion of the ether fraction was evaporated to dryness and dissolved in $100 \mu \mathrm{l}$ of methanol. Detection and quantification of polyamines were performed with a Shimadzu LC-6A high-performance liquid chromatograph; a Zorbax octadecylsilane column $(4.6 \mathrm{~mm}$ by $15 \mathrm{~cm}$; particle size $5 \mu \mathrm{m}$ ) (Dupont Co., Kyoto, Japan) with a mobile phase of $60 \%$ aqueous methanol, a flow rate of $1 \mathrm{ml} / \mathrm{min}$, and a detection wavelength of $254 \mathrm{~nm}$ was used. The calculation of the polyamine levels was based on the average area counts of each of the three standard polyamines.

\section{RESULTS}

Effect of polyamines and MGBG on the prepenetration morphogenesis in C. miyabeanus. Appressorium formation in 
C. miyabeanus was inhibited by putrescine, spermidine, and spermine in a dose-dependent manner (Figs. 1 and 2). Spermidine and spermine specifically inhibited appressorium formation $\approx 67$ and $83 \%$ at $10 \mathrm{mM}$, respectively. These polyamines did not affect conidial germination even at a higher concentration. Putrescine inhibited appressorium formation $\approx 86 \%$ at $1 \mathrm{mM}$; however, it also abolished conidial germination at 10 and $50 \mathrm{mM}$. In addition, conidial germination and appressorium formation were completely abolished by the SAM decarboxylase inhibitor, MGBG, in all testing concentration.

Calcium restores appressorium formation inhibited by MGBG. Curtailment of appressorium formation by putrescine, spermidine, or spermine on the hydrophobic surface of GelBond was not recovered by $1 \mathrm{mM}$ cyclic AMP (cAMP), 1,16-hexadecanediol, or $\mathrm{CaCl}_{2}$ (data not shown). Although cAMP and 1,16hexadecanediol did not affect inhibitory effects of MGBG on prepenetration development, exogenous calcium nullified MGBG effects on the hydrophobic surface of GelBond (Fig. 2).

Effect of MGBG and calcium on the conidial germination and appressorium formation in vivo. To evaluate the effect of MGBG and calcium on the prepenetration morphogenesis on rice leaves, drops of conidial suspension along with MGBG or $\mathrm{CaCl}_{2}$ were placed onto detached rice leaves and conidial development was observed. MGBG reduced the number of attached conidia and appressoria. For more clear observation, chlorophyll was removed and then fungal cells were stained. Twelve hours after placement, conidia germinated and formed appressoria on rice leaves (Fig. 2). MGBG inhibited conidial germination and appressorium formation $\approx 31$ and $90 \%$, respectively. However, calcium treatment restored both conidial germination and appressorium formation.
Effects of MGBG and $\mathrm{CaCl}_{2}$ on the disease progression. We also evaluated the effects of MGBG and $\mathrm{CaCl}_{2}$ on the progression of rice brown leaf spot in the greenhouse. Spraying MGBG on rice did not cause any obvious phytotoxic symptoms for up to 14 days. Control plants treated with a solution of Tween 20 at $250 \mu \mathrm{g} / \mathrm{ml}$ followed by inoculation with the pathogen $24 \mathrm{~h}$ later resulted in typical brown leaf spot symptoms and disease severity of 8 was reached $\approx 4$ days after inoculation (Fig. 3). Treatment of plants with MGBG $24 \mathrm{~h}$ before inoculation significantly reduced the number of lesions; however, the lesion size was not affected by MGBG. Progression of disease was restored by the synchronous treatment of $1 \mathrm{mM} \mathrm{CaCl}$, with conidial suspension on the rice leaves pretreated with MGBG.

Transition of polyamines. Freshly collected conidia of C. miyabeanus contained a large amount of polyamines. The quantity of polyamines within fungal conidia decreased during conidial germination or appressorium formation (Fig. 4). Levels of spermidine decreased significantly during these developments, whereas those of putrescine and spermine remained unchanged. The MGBG treatment further reduced the amount of polyamines within germinating or appressorium-forming conidia and inhibited specific decrease of spermidine levels. In addition, these variations were not restored by the exogenous calcium treatment.

Calcium restores calmodulin $(\mathrm{Cm} \mathrm{CaM})$ gene expression and PKC activity inhibited by MGBG. Effects of MGBG and exogenous calcium on the transcription of the calmodulin gene were investigated and are shown in Figure 5A. Messenger RNA (mRNA) of $C m C a M$ accumulated at a very low level within conidia. At $4 \mathrm{~h}$ after the hard surface contact, $\mathrm{CmCaM}$ transcribed highly within germinating or appressorium-forming conidia. MGBG inhibited the CmCaM transcription and calcium com-
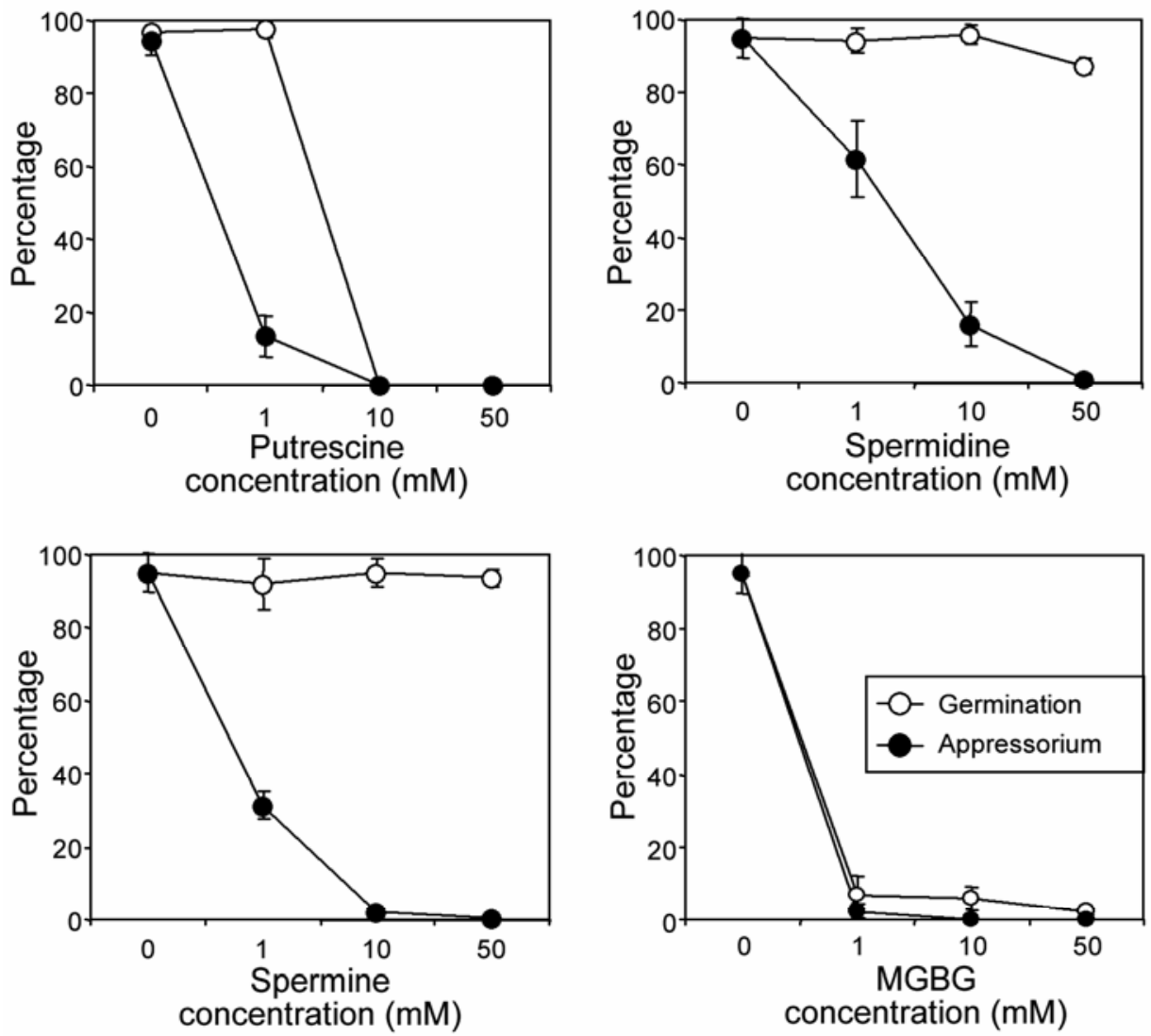

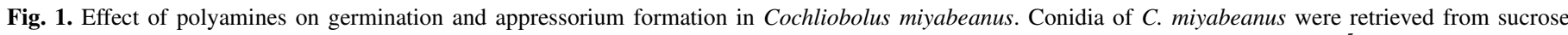

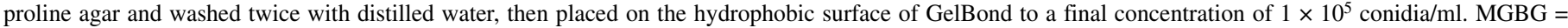

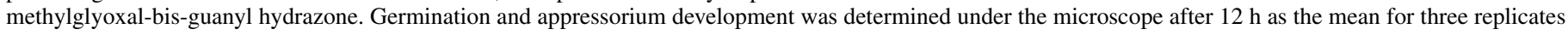
of 100 conidia each. Each point represents the mean \pm standard error. 
pletely restored mRNA synthesis of $\mathrm{CmCaM}$ curtailed by MGBG. To further investigate the relation between polyamine biosynthesis and calcium, PKC activity was estimated because PKC target substrates include calcium- and calmodulin-binding proteins. Only a barely detectable level of PKC activity was measured in conidia. Fortified PKC activity was observed within germinating or appressorium-forming conidia (Fig. 5B). MGBG also inhibited this enhanced PKC activity and calcium restored PKC activity interdicted by MGBG.

\section{DISCUSSION}

Our results demonstrated that all the polyamines abolished appressorium formation in C. miyabeanus. An inhibitor of polyamine biosynthesis, MGBG, also inhibited appressorium formation. Therefore, proper regulation of intracellular polyamine con- centration should be essential for prepenetration morphogenesis of this fungus. Polyamines and MGBG specifically inhibited appressorium formation in the rice blast fungus, $M$. grisea, whereas germination remained unaffected (9). Similarly, spermidine and spermine in this study specifically inhibited appressorium formation in C. miyabeanus. On the other hand, putrescine and MGBG inhibited conidial germination and appressorium formation in $C$. miyabeanus. Taken together, these differences suggest that there are species-specific variances among polyamine-biosynthetic pathways or polyamine-related signaling cascades culminating in germination and appressorial development.

In the detached-leaf assay, greatly reduced numbers of conidia and appressoria were observed on rice leaves treated with MGBG. Due to the vigorous nature of the process of chlorophyll removal and staining procedure of fungal cells, conidia without
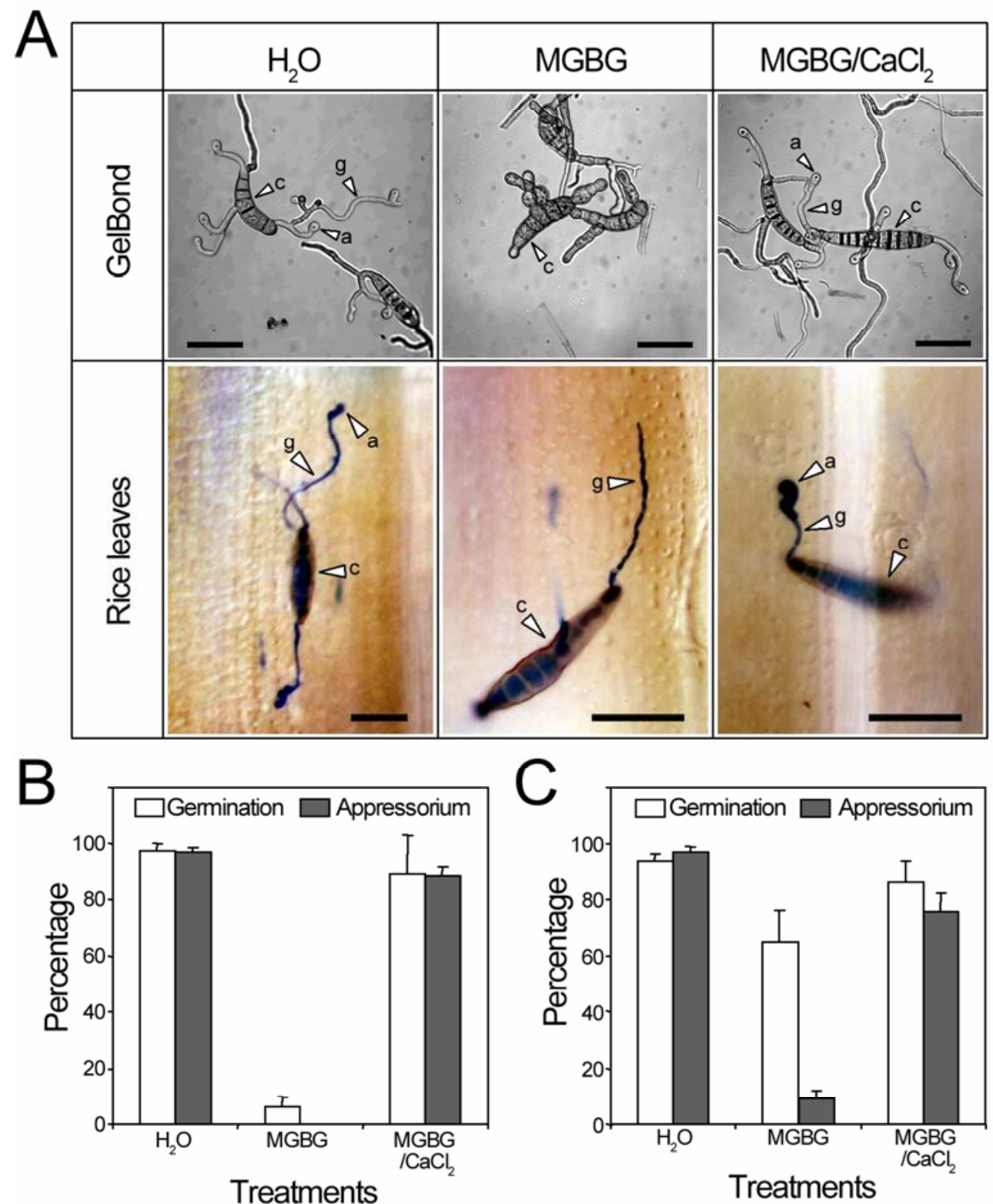

Fig. 2. Effects of methylglyoxal-bis-guanyl hydrazone (MGBG) or $\mathrm{CaCl}_{2}$ on conidial germination and appressorium formation of Cochliobolus miyabeanus in vitro and in vivo. A, Effects of MGBG or $\mathrm{CaCl}_{2}$ on prepenetration morphogenesis of $C$. miyabeanus on the hydrophobic surface of GelBond and on the rice leaves. Conidial germination and appressorium formation inhibited by MGBG were restored by exogenous calcium. Conidia of $C$. miyabeanus were retrieved from sucrose proline agar, washed twice with distilled water, and placed on the hydrophobic surface of GelBond or rice leaves with $1 \mathrm{mM} \mathrm{MGBG}$ or $1 \mathrm{mM} \mathrm{CaCl} \mathrm{C}_{2}$ to a final concentration of $1 \times 10^{5}$ and $1 \times 10^{4}$ conidia/ml. Bars $=50 \mu \mathrm{m}, \mathrm{a}=$ appressorium, $\mathrm{c}=$ conidium, and $\mathrm{g}=$ germ tube. $\mathbf{B}$, Effects of MGBG or CaCl $\mathrm{M}_{2}$ on prepenetration morphogenesis of C. miyabeanus on the hydrophobic surface of GelBond. $\mathbf{C}$, Effects of $\mathrm{MGBG}$ or $\mathrm{CaCl}_{2}$ on prepenetration morphogenesis of C. miyabeanus on the rice leaves. Conidial germination and appressorium formation were determined under the microscope after $12 \mathrm{~h}$ as the mean for three replicates of 100 conidia each. Each bar represents the mean and vertical lines on top of bars + half standard error. 
appressoria might have been shaken off. Conidial germination and appressorium formation progressed successfully on the artificial surface and on the rice leaves. Intriguingly, effects of MGBG on prepenetration morphogenesis in vitro and in vivo were distinct. On the artificial surface, both developments were nearly completely inhibited by MGBG. Appressorium formation also was inhibited by MGBG on rice leaves; however, curtailment of the conidial germination was not severe compared with that on the hydrophobic surface of GelBond. These results indicate that certain plant surface factors partly compensate the disturbance of polyamine metabolism by MGBG amendment and this restoration is not effective on appressorium formation but on conidial germination in vivo. Up-to-date chemical cues affecting preinfection morphogenesis have been investigated. One of the well-known pathogens, $M$. grisea, germinates and forms appressorium by sensing the cutin monomer within rice surface, 1,16-hexadecanediol (17). Host chemical signals, waxes, and ethylene also trigger germination and appressorium formation in Colletotrichum gloeosporioides infecting avocado (14). To further investigate the chemical cues responsible for the recovery of conidial germination abolished by MGBG on the GelBond, we isolated plant surface wax using chloroform, evaporated the solvent, and resuspended the wax in methanol. In addition, effects

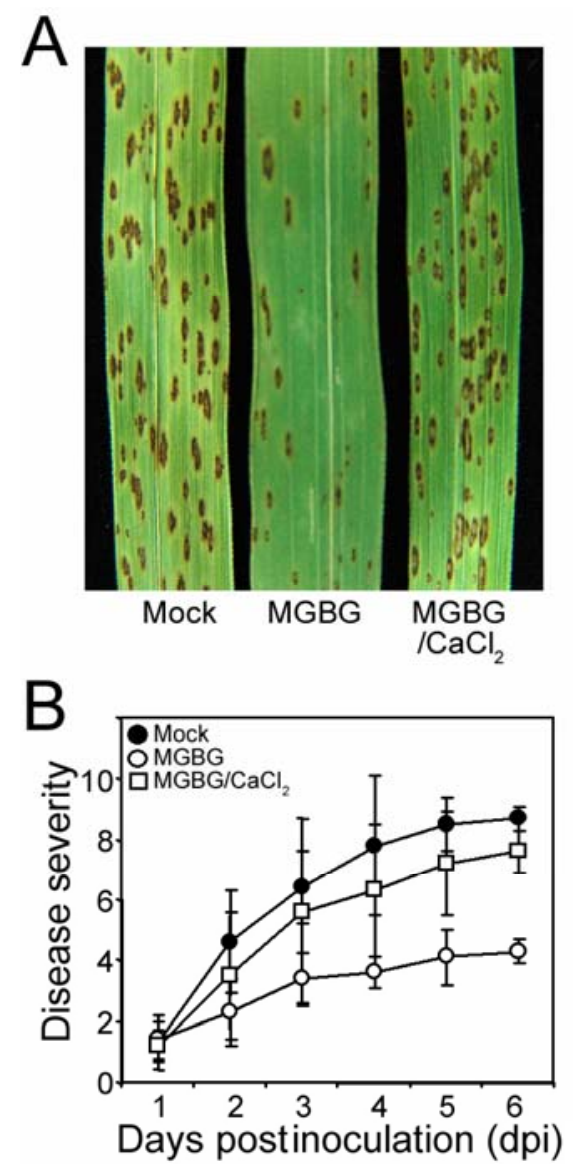

Fig. 3. Effects of methylglyoxal-bis-guanyl hydrazone (MGBG) and $\mathrm{CaCl}_{2}$ on the progression of rice brown leaf spot. MGBG $(1 \mathrm{mM})$ supplemented with Tween 20 at $250 \mu \mathrm{g} / \mathrm{ml}$ (mock) or mock was sprayed on rice leaves and air dried. Conidial suspension of Cochliobolus miyabeanus supplemented with mock or $1 \mathrm{mM} \mathrm{CaCl}_{2}$ with mock was inoculated. A, MGBG significantly reduced the progression of rice brown leaf spot and $\mathrm{CaCl}_{2}$ treatment with conidial suspension nullified reduction of disease by MGBG. Leaves were harvested 7 days after inoculation and photographed. B, Inhibition of rice brown leaf spot by MGBG and nullification of MGBG effect by calcium treatment. Rating is based on the proportion of symptomatic leaf area as described in Materials and Methods. All experiments were conducted three times independently, with three replicates of 10 rice plants. Each point represents the mean \pm standard error. of cutin monomer also were examined. The cutin monomer and rice surface wax did not affect conidial germination and appressorium formation abolished by MGBG on the GelBond (data not shown). Therefore, the cutin monomer and plant surface wax are not plant factors compensating inhibitory effects of MGBG on the conidial germination. It also may be possible that the extraction procedure was not suitable or the active component within rice surface was volatile or was unstable; thus, biological activity had been lost. Although precise biochemical processes remain to be elucidated, our results indicate that environmental cues within the rice surface other than thigmo-signals affect polyamine biosynthesis or polyamine-mediated molecular and biochemical responses indispensable for prepenetration morphogenesis in Cochliobolus miyabeanus.

To confirm these results further, effects of $\mathrm{MGBG}$ or $\mathrm{CaCl}_{2}$ on the progression of rice brown leaf spot were investigated. MGBG distinctively reduced disease severity of rice brown leaf spot and exogenous $\mathrm{CaCl}_{2}$ restored progression of disease suppressed by MGBG. Interestingly, MGBG treatment diminished the numbers of lesions and the lesion size did not vary. These results indicate that MGBG has little effect on the fungal ramification in planta. In several host species, polyamine biosynthesis inhibitors such as DFMO and MGBG successfully inhibited fungal diseases via inhibiting appressorium formation (37).

To dissect the in vitro effects of MGBG and exogenous $\mathrm{CaCl}_{2}$ more clearly, the amounts of developing polyamines within freshly collected conidia and conidia were estimated. Similarly to our results, high levels of polyamines were detected within freshly collected conidia of $M$. grisea (9). However, putrescine and spermidine are major polyamines in conidia of $C$. miyabeanus and those in $M$. grisea are spermidine and spermine. In addition, the polyamine level decreased rapidly during conidial germination of both fungi. The putrescine/spermidine ratio was raised during conidial germination in both fungi because of the distinctive decrease of spermidine levels. A similar decrease was observed in the spore of Aspergillus flavus during microcyclic differentiation (28). MGBG treatment fortified decrease of poly-

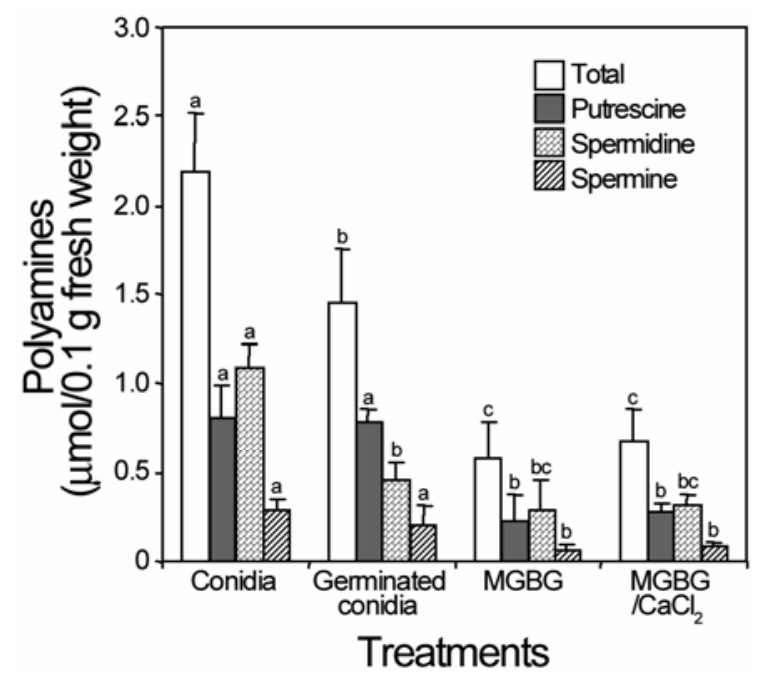

Fig. 4. Effects of methylglyoxal-bis-guanyl hydrazone (MGBG) or $\mathrm{CaCl}_{2}$ on the level of polyamines within conidia of Cochliobolus miyabeanus. Polyamines were isolated from each $100 \mathrm{mg}$ of freshly collected conidia, germinating or appressorium-forming conidia, germinating or appressorium-forming conidia treated with $1 \mathrm{mM}$ MGBG, and germinating or appressorium-forming conidia treated with $1 \mathrm{mM} \mathrm{MGBG}$ and $1 \mathrm{mM} \mathrm{CaCl}_{2}$. Prior to placement on the glass plate, $1 \mathrm{mM}$ MGBG or $1 \mathrm{mM} \mathrm{CaCl}_{2}$ was mixed with conidial suspension and germinating or appressorium-forming conidia were recovered $4 \mathrm{~h}$ after treatments. The experiments were repeated three times independently with three replicates. Each bar represents the mean + half standard error. Different letters indicate statistically significant differences between treatments (Duncan's multiple range test, $P=0.05)$. 
amine levels and inhibited increment of the putrescine/spermidine ratio. MGBG supplementation also reduced polyamines in pine callus (38). These results further support our hypothesis that finetuning of polyamine transition is required for the normal prepenetration morphogenesis in C. miyabeanus. Proper regulation of polyamine transition is essential for sporulation and conidial germination in A. nidulans (25) and sclerotial formation in Sclerotinia sclerotiorum (34).

Exogenous calcium restored prepenetration morphogenesis and pathogenicity and did not affect MGBG effects on the polyamine transition. To investigate the relationship between polyamine metabolism and $\mathrm{Ca}^{2+}$-dependent signaling pathways, CmCaM transcription, and PKC activity were assessed in the presence of MGBG or exogenous calcium. Calmodulin, one of the most ubiquitous and conserved calcium-binding proteins, plays a major role on conidial germination and appressorium formation in several plant-pathogenic fungi (31). PKCs phosphorylate numerous proteins participate in cell signaling, including calcium- and calmodulin-binding proteins (20). Transcription of $\mathrm{CmCaM}$ and PKC activity were significantly upregulated in germinating or appressorium-forming conidia of $C$. miyabeanus in this analysis. Further, MGBG suppressed $\mathrm{CmCaM}$ expression and PKC activity within germinating conidia and exogenous calcium restored both molecular and biochemical responses observed in normal development. These results strongly suggest that inhibition of $\mathrm{CmCaM}$ transcription and downregulation of $\mathrm{PKC}$ are $\mathrm{MGBG}$ functions
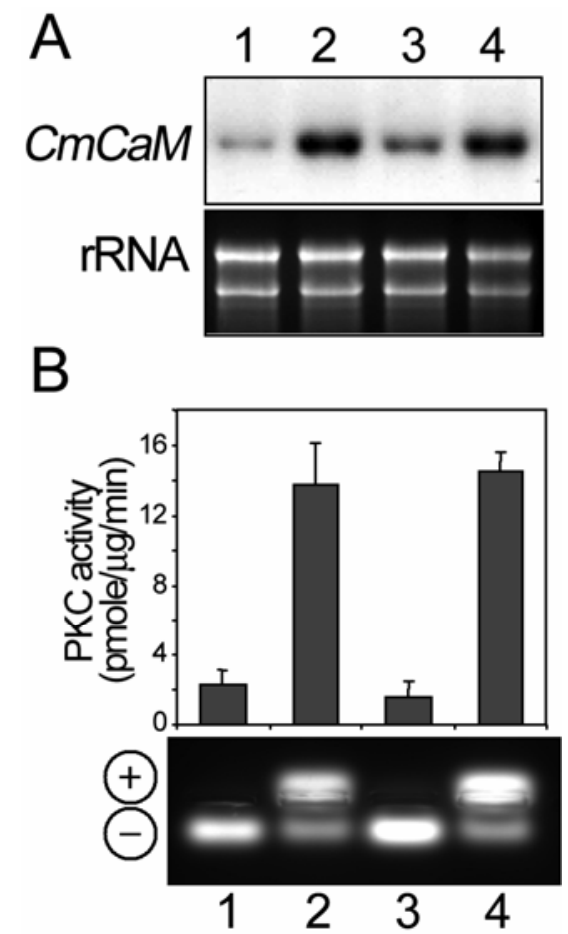

Fig. 5. Effects of methylglyoxal-bis-guanyl hydrazone (MGBG) and $\mathrm{CaCl}_{2}$ on the expression of calmodulin $(\mathrm{CmCaM})$ gene and protein kinase $\mathrm{C}$ ( $\mathrm{PKC}$ ) activity during prepenetration morphogenesis in Cochliobolus miyabeanus. Prior to placement on the glass plate, $1 \mathrm{mM}$ MGBG or $1 \mathrm{mM} \mathrm{CaCl}_{2}$ was mixed with conidial suspension and the germinated conidia were recovered $4 \mathrm{~h}$ after treatments. A, Total RNAs $(5 \mu \mathrm{g})$ from conidia (lane 1$)$, hard surfaceresting conidia (lane 2), hard surface-resting conidia with $1 \mathrm{mM}$ MGBG (lane 3 ), and hard surface-resting conidia with $1 \mathrm{mM} \mathrm{CaCl}_{2}$ and $1 \mathrm{mM} \mathrm{MGBG}$ (lane 4) were subjected to Northern blot analysis with calmodulin complementary DNA $(\mathrm{CmCaM})$ as the probe. Equal sample loading was confirmed by ethidium bromide staining of the rRNA in the gel. B, MGBG inhibited PKC activity in germinated conidia and $\mathrm{CaCl}_{2}$ restored $\mathrm{PKC}$ activity inhibited by MGBG. Total proteins $(10 \mu \mathrm{g})$ from conidia (lane 1), hard surface-resting conidia (lane 2), hard surface-resting conidia with $1 \mathrm{mM} \mathrm{MGBG}$ (lane 3), and hard surface-resting conidia with $1 \mathrm{mM} \mathrm{CaCl}_{2}$ and $1 \mathrm{mM} \mathrm{MGBG}$ (lane 4) were subjected to PKC assay. All experiments were conducted with three replicates and each point represents the mean + half standard error. during conidial germination and appressorium formation in C. miyabeanus. Accumulation of calmodulin mRNA during conidial germination and appressorium formation has been drawn in $M$. grisea (31), Colletotrichum gloeosporioides virulent on avocado (29) and red pepper (1), and C. trifolii (45). In addition, disturbance of the calcium- or calmodulin-dependent signaling pathway and protein phosphorylation by polyamines have been described previously in the mammalian gland cells (11) and C. gloeosporioides infecting red pepper (1). In summary, acting sites of MGBG in the prepenetration morphogenesis of Cochliobolus miyabeanus are upstream of where $\mathrm{Ca}^{2+}$ functions and suggests that MGBG may disturb proper regulation of intracellular $\mathrm{Ca}^{2+}$ levels. Several reports also suggest the close relationship between polyamine metabolism and calcium-mediated signaling pathways $(8,18)$.

Although the precise mechanisms still remain unclear, results presented here suggest that MGBG extends inhibitory effects through $\mathrm{Ca}^{2+}$ - or calmodulin- and PKC-dependent signaling pathways in $C$. miyabeanus infecting rice. Unveiling the specific interaction between polyamines and $\mathrm{Ca}^{2+}$ or calmodulin-dependent signaling pathways with respect to fungal cell developments is not only of interest in cell biology but also important in providing direction for the development of novel strategies to control rice brown leaf spot.

\section{ACKNOWLEDGMENTS}

This research was supported by a grant from the National Institute of Agricultural Biology to I.-P. Ahn and a grant (CG2211) from the Crop Functional Genomics Center of the 21st Century Frontier Research Program funded by the Ministry of Science and Technology and Rural Development Administration of the Korean government to S.-C. Suh.

\section{LITERATURE CITED}

1. Ahn, I.-P., Kim, S., Choi, W.-B., and Lee, Y.-H. 2003. Calcium restores prepenetration morphogenesis abolished by polyamines in Colletotrichum gloeosporioides infecting red pepper. FEMS Microbiol. Lett. 227:237241.

2. Ahn, I.-P., Uhm, K.-H., Kim, S., and Lee, Y.-H. 2003. Signaling pathways involved in preinfection development of Colletotrichum gloeosporioides, C. coccodes, and C. dematium pathogenic on red pepper. Physiol. Mol. Plant Pathol. 63:281-289.

3. Antognoni, F., Agostani, S., Spinelli, C., Koskinen, M., Elo, H., and Bagni, N. 1999. Effect of bis(guanylhydrazones) on growth and polyamine uptake in plant cells. J. Plant Growth Regul. 18:39-44.

4. Azzi, A., Boscoboinik, D., and Hensey, C. 1992. The protein kinase C family. Eur. J. Biochem. 208:547-557.

5. Bailey, A., Mueller, E., and Bowyer, P. 2000. Ornithine decarboxylase of Stagonospora (Septoria) nodorum is required for virulence toward wheat. J. Biol. Chem. 275:14242-14247.

6. Bradford, M. M. 1976. A rapid and sensitive method for the quantitation of microgram quantities of protein utilizing the principle of protein-dye binding. Anal. Biochem. 72:248-254.

7. Braun, E. J., and Howard, R. J. 1994. Adhesion of Cochliobolus heterostrophus conidia and germlings to leaves and artificial surfaces. Exp. Mycol. 18:211-220.

8. Chang, B. K. 1991. Inhibitory effects of a calcium antagonist on ornithine decarboxylase induction in pancreatic cancer cell lines. Pancreas 631-636.

9. Choi, W.-B., Kang, S.-H., Lee, Y.-W., and Lee, Y.-H. 1998. Cyclic AMP restores appressorium formation inhibited by polyamines in Magnaporthe grisea. Phytopathology 88:58-62.

10. Clay, R. P., Bergmann, C. W., and Fuller, M. S. 1997. Isolation and characterization of an endopolygalacturonase from Cochliobolus sativus and a cytological study of fungal penetration of barley. Phytopathology 87:1148-1159.

11. Combest, W. L., and Gilbert, L. I. 1992. Polyamines modulate multiple protein phosphorylation pathways in the insect prothoracic gland. Mol. Cell Endocrinol. 83:11-19.

12. Degani, O., Maor, R., Hadar, R., Sharon, A., and Horwitz, B. A. 2004. Host physiology and pathogenic variation of Cochliobolus heterostrophus strains with mutations in the $\mathrm{G}$ protein alpha subunit, CGA1. Appl. Environ. Microbiol. 70:5005-5009.

13. Dhingra, O. D., and Sinclair, J. B. 1985. Basic Plant Pathology Methods. CRC Press, Boca Raton, FL. 
14. Flaishman, M. A., Hwang, C.-H., and Kolattukudy, P. E. 1995. Involvement of protein phosphorylation in the induction of appressorium formation in Colletotrichum gloeosporioides by its host surface wax and ethylene. Physiol. Mol. Plant Pathol. 47:103-117.

15. Flores, H. E., and Galston, A. W. 1982. Analysis of polyamines in higher plants by high performance liquid chromatography. Plant Physiol. 69:701706.

16. Ganem, S., Lu, S.-W., Lee, B.-N., Chou, D. Y.-T., Hadar, R., Turgeon, B. G., and Horwitz, B. A. 2004. G-protein b-subunit of Cochliobolus heterostrophus involved in virulence, asexual and sexual reproductive ability, and morphogenesis. Eukaryotic Cell 3:1653-1663.

17. Gilbert, R. D., Johnson, A. M., and Dean, R. A. 1996. Chemical signals responsible for appressorium formation in the rice blast fungus Magnaporthe grisea. Physiol. Mol. Plant Pathol. 48:335-346.

18. Gomez, M., and Hellstrand, P. 1999. Endogenous polyamines modulate $\mathrm{Ca}^{2+}$ channel activity in guinea-pig intestinal smooth muscle. Eur. J. Physiol. 438:445-451.

19. Guevara-Olvera, L., Xoconostle-Cazares, B., and Ruiz-Herrera, J. 1997. Cloning and disruption of the ornithine decarboxylase gene of Ustilago maydis: Evidence for a role of polyamines in its dimorphic transition. Microbiology 143:2237-2245.

20. Heinisch, J. J., Lorberg, A., Schmitz, H.-P., and Jacoby, J. J. 1999. The protein kinase $\mathrm{C}$-mediated MAP kinase pathway involved in the maintenance of cellular integrity in Saccharomyces cerevisiae. Mol. Microbiol. 32:671-680.

21. Horwitz, B. A., Sharon, A., Lu, S. W., Ritter, V., Sandrock, T. M., Yoder, O. C., and Turgeon, B. G. 1999. A G protein alpha subunit from Cochliobolus heterostrophus involved in mating and appressorium formation. Fungal Genet. Biol. 26:19-32.

22. International Rice Research Institute. 1988. Standard Evaluation System for Rice, 3rd ed. International Rice Testing Program, International Rice Research Institute, Los Baños, The Philippines.

23. Jackson, A. J., Walters, D. R., and Marshall, G. 1994. Evaluation of Penicillium chrysogenum and its antifungal extracts as potential biological control agents against Botrytis fabae on faba beans. Mycol. Res. 98:11171126.

24. Jambunathan, N., and McNellis, T. W. 2003. Regulation of Arabidopsis COPINE 1 gene expression in response to pathogens and abiotic stimuli. Plant Physiol. 132:1370-1381.

25. Jin, Y., Bok, J. W., Guzman-de-Pena, D., and Keller, N. P. 2002. Requirement of spermidine for developmental transitions in Aspergillus nidulans. Mol. Microbiol. 46:801-812.

26. Kang, S. H., Khang, C. H., and Lee, Y.-H. 1999. Regulation of cAMPdependent protein kinase during appressorium formation in Magnaporthe grisea. FEMS Microbiol. Lett. 170:419-423.

27. Kardin, M. K., and Percich, J. A. 1983. Resistance of Bipolaris oryzae to fenapanil. Plant Dis. 67:871-874.

28. Khurana, N., Saxena, R., Gupta, R., and Rajam, M. 1996. Polyamines as modulators of microcycle conidiation in Aspergillus flavus. Microbiology $142: 517-523$

29. Kim, Y. K., Li, D., and Kolattukudy, P. E. 1998. Induction of $\mathrm{Ca}^{2+}$ calmodulin signaling by hard-surface contact primes Colletotrichum gloeosporioides conidia to germinate and form appressoria. J. Bacteriol. 180:5144-5150.

30. Lee, S.-C., and Lee, Y.-H. 1998. Calcium/calmodulin-dependent signaling for appressorium formation in the plant pathogenic fungus Magnaporthe grisea. Mol. Cells 6:698-704.

31. Liu, Z. M., and Kolattukudy, P. E. 1999. Early expression of the calmodulin gene, which precedes appressorium formation in Magnaporthe grisea, is inhibited by self-inhibitors and requires surface attachment. J. Bacteriol. 181:3571-3577.

32. Marton, L. J., and Morris, D. R. 1987. Inhibition of polyamine biosynthesis. Pages 79-105 in: Biological Significance and Basis for New Therapies. Academic Press, New York.

33. Ou, S. H. 1985. Rice Diseases, 2nd ed. Commonwealth Mycological Institute, Kew, England.

34. Pieckenstain, F., Garriz, A., Chornomaz, E., Sanchez, D., and Ruiz, O. 2001. The effect of polyamine biosynthesis inhibition on growth and differentiation of the phytopathogenic fungus Sclerotinia sclerotiorum. Antonie Leeuwenhoek 80:245-253.

35. Pollard, K. J., Samuels, M. L., Crowley, K. A., Hansen, J. C., and Peterson, C. L. 1999. Functional interactions between GCN5 and polyamines: A new role for core histone acetylation. EMBO J. 18:56225633.

36. Rajam, M. V., Weinstein, L. H., and Galston, A. W. 1985. Prevention of a plant disease by specific inhibition of fungal polyamine biosynthesis. Proc. Natl. Acad. Sci. USA 82:6874-6878.

37. Reitz, M., Walters, D., and Moerschbacher, B. 1995. Germination and appressorial formation by uredospores of Uromyces viciae-fabae exposed to inhibitors of polyamine. Eur. J. Plant Pathol. 101:573-578.

38. Sarjala, T., Haggman, H., and Aronen, T. 1997. Effect of exogenous polyamines and inhibitors of polyamine biosynthesis on growth and free polyamine contents of embryogenic Scots pine callus. J. Plant Physiol. 150:597-602.

39. Stuthman, D. D. 2002. Contribution of durable disease resistance to sustainable agriculture. Euphytica 124:253-258.

40. Tabor, C. W., and Tabor, H. 1985. Polyamines in microorganisms. Microbiol. Rev. 49:81-99.

41. Uhm, K.-H., Ahn, I.-P., Kim, S., and Lee, Y.-H. 2003. Calcium/ calmodulin-dependent signaling for prepenetration development in Colletotrichum gloeosporioides. Phytopathology 93:82-87.

42. Walters, D. R. 1995. Inhibition of polyamine biosynthesis in fungi. Mycol. Res. 99:129-139.

43. Walters, D. R., Keenan, J. P., Cowley, T., McPherson, A., and Havis, N. D. 1995. Inhibition of polyamine biosynthesis in Phytophthora infestans and Pythium ultimum. Plant Pathol. 44:80-85.

44. Warwar, V., and Dickman, M. B. 1996. Effects of calcium and calmodulin on spore germination and appressorium development in Colletotrichum trifolii. Appl. Environ. Microbiol. 62:74-79.

45. Warwar, V., Oved, S., and Dickman, M. B. 2000. Antisense expression of the calmodulin gene from Colletotrichum trifolii impairs prepenetration development. FEMS Microbiol. Lett. 191:213-219. 